\title{
Funktionserholung nach Schlaganfall und die therapeutische Rolle der nicht-invasiven Hirnstimulation
}

\section{Functional Recovery after Stroke and the Therapeutic Relevance of Non-invasive Brain Stimulation}

\author{
Autoren \\ Caroline Tscherpel1, 2 , Christian Grefkes ${ }^{1,2}$ \\ Institute \\ 1 Klinik und Poliklinik für Neurologie, Universitätsklinik \\ Köln \\ 2 Institut für Neurowissenschaften und Medizin (INM-3), \\ Forschungszentrum Jülich
}

Schlüsselwörter

Exzitabilität, Neurorehabiltiation, Plastizität, Neuromodulation, Hirnstimulation

Key words

Excitability, neurorehabilitation, plasticity, neuromodulation, brain stimulation

online publiziert $\quad 29.10 .2020$

Bibliografie

Klin Neurophysiol 2020; 51: 214-223

DOI 10.1055/a-1272-9435

ISSN 1434-0275

(c) 2020. Thieme. All rights reserved.

Georg Thieme Verlag KG, Rüdigerstraße 14,

70469 Stuttgart, Germany

Korrespondenzadresse

Prof. Dr. Christian Grefkes

Klinik und Poliklinik für Neurologie

Universitätsklinik Köln

Kerpener Straße 62

50937 Köln

Deutschland

christian.grefkes@uk-koeln.de

\section{ZUSAMMENFASSUNG}

Im Bereich der non-invasiven Hirnstimulation stellen die transkranielle Magnetstimulation (engl. transcranial magnetic stimulation, TMS) sowie die transkranielle Gleichstromstimulation (engl. transcranial direct current stimulation, tDCS) bis heute die wichtigsten Techniken zur Modulation kortikaler Erregbarkeit dar. Beide Verfahren induzieren Nacheffekte, welche die Zeit der reinen Stimulation überdauern, und ebnen damit den Weg für ihren therapeutischen Einsatz beim Schlaganfall. In diesem Übersichtsartikel diskutieren wir die aktuelle Datenlage TMS- und tDCS-vermittelter Therapien für die häufigsten schlaganfallbedingten Defizite wie Hemiparese, Aphasie und Neglect. Darüber hinaus adressieren wir mögliche Einschränkungen der gegenwärtigen Ansätze und zeigen Ansatzpunkte auf, um Neuromodulation nach Schlaganfall effektiver zu gestalten und damit das Outcome der Patienten zu verbessern.

\section{ABSTRACT}

Transcranial magnetic stimulation (TMS) and transcranial direct current stimulation (tDCS) currently represent the most important techniques for modulating cortical excitability. Both approaches induce after-effects that outlast the time of stimulation, and thus form the foundation for their therapeutic use in stroke rehabilitation. The purpose of this review is to discuss current data on non-invasive stimulation therapies for the most common stroke-related deficits, i. e., hemiparesis, aphasia and neglect. In addition, we address current limitations and finally suggest strategies to potentially increase the effectiveness of neuromodulation after stroke in order to improve patients' outcome.

\section{Einleitung}

Die Erholung nach einem Schlaganfall umfasst viele Dimensionen: Auf der Ebene einzelner Zellen stößt eine Kaskade molekularer und biochemischer Mechanismen die Neuvernetzung von Neuronen mit Wachstum des Dendritenbaums, Sprossung von Axonen sowie der Ausbildung neuer Synapsen an und formt damit die Grundlage von Neuroplastizität [1-4]. Auf der Netzwerkebene können Erholungsprozesse nicht nur zu Veränderungen der Aktivität und Konnektivität um die Schlaganfallläsion herum, sondern zur Umgestal- tungen der funktionellen Netzwerkstruktur beider Hemisphären führen [5-8]. Auf funktioneller Ebene stellt die Erholung von schlaganfallbedingten Defizite eine Kombination spontaner und lernabhängiger Prozesse dar $[9,10]$. Diese reichen von Restitution, d. h. dem vollumfänglichen Wiedergewinn der Funktionalität vor dem Ereignis [11, 12], über Substitution und Kompensation, welche die neuronale Reorganisation zum Wiedererlernen verlorengegangener Funktionen und Anpassung an die beeinträchtigten Fähigkeiten eines Patienten einschließen [9, 12]. 
Die Zeitabhängigkeit der neurologischen Erholung nach einem Schlaganfall wurde in Längsschnittstudien wiederholt über die verschiedenen funktionellen Systeme der Motorik, Sprache und des Aufmerksamkeitssystems hinweg gezeigt [13-16]. So treten die größten Verbesserungen innerhalb der ersten Wochen nach dem Ereignis auf - ein kritischer Zeitraum, der in tierexperimentellen Studien durch erhöhte Neuroplastizität gekennzeichnet ist [17-20] - und erreichen anschließend in der chronischen Phase nach 6-12 Monaten ein relativ stabiles Plateau mit in der Regel nur noch marginalem zusätzlichen Funktionszugewinn [9, 21-23].

Jede der genannten Dimensionen der Erholung ist jedoch geprägt von einer frappierenden, interindividuellen Variabilität [9, 21-23]. Während offensichtliche Einflussfaktoren wie Alter, Komorbiditäten, Grad der Betroffenheit sowie Läsionsgröße und -lokalisation relativ gut verstanden sind [21,23-25], fehlt bislang noch ein tiefgreifendes Verständnis der neuronalen Veränderungen sowie der sich anschließenden Reorganisationsprozesse nach einem Schlaganfall auf der individuellen Ebene. In anderen Worten: Es ist weiterhin unklar, warum sich der eine Patient relativ gut erholt, der andere Patient mit ähnlichem Defizit und Läsionslokalisation jedoch nicht. Die Kenntnis um diese Prozesse scheint jedoch eine entscheidende Voraussetzung zu sein, um Funktionserholung effektiv zu fördern.

\section{Non-invasive Hirnstimulation und ihre zugrundeliegenden Konzepte}

Bildgebende Verfahren und insbesondere die funktionelle Magnetresonanztomographie (fMRT) haben das aktuelle Verständnis der neuronalen Reorganisationsmechanismen nach einem Schlaganfall entscheidend geprägt. Eine konsistente Beobachtung über die funktionellen Domänen hinweg sind weitreichende Veränderungen kortikaler Aktivitätsmuster sowohl in der betroffenen (ipsiläsionellen) als auch in der intakten (kontraläsionellen) Hemisphäre [5-7]. Ist die akute Phase nach einem Schlaganfall durch einen initialen Abfall der neuronalen Aktivität in der ipsiläsionellen Hemisphäre gekennzeichnet, so folgt demgegenüber innerhalb der ersten Wochen eine Aktivitätssteigerung sowohl in der betroffenen als auch in der kontraläsionellen Hemisphäre [5, 8, 26]. Während es für diese Zeit noch Hinweise gibt, dass die Aktivitätszunahme mit funktioneller Erholung in positivem Zusammenhang steht $[5,27,28]$, ist eine anhaltende, kontraläsionelle Aktivitätssteigerung in der chronischen Phase mit einem schlechten funktionellen Outcome verbunden [5, 26, 29, 30].

Diese gegensätzlichen Ergebnisse haben 2 Modelle der funktionellen Bedeutung der Aktivitätsveränderungen geprägt und sind auch aktuell noch Gegenstand der Forschung sowie wissenschaftlicher Debatten [31, 32]. So könnte die Überaktivität nicht betroffener Areale einerseits im Rahmen der Vikariationstheorie (vicarius [lat.]: Stellvertreter) einen unterstützenden Mechanismus darstellen, indem die Funktion der von der ischämischen Läsion ge-/ zerstörten Regionen von intakten Arealen übernommen wird. Alternativ prägen Befunde aus Konnektivitätsanalysen [33] sowie Untersuchungen, welche mittels transkranieller Magnetstimulation (TMS) erhoben wurden, das Modell der maladaptiven interhemisphärischen Kompetition nach einem Schlaganfall. Hier wird Über- aktivität als störender Faktor gewertet, welche die physiologische Funktion der normalerweise für die Durchführung einer Aufgabe beteiligten Hirnregionen kompromittiert. Beispielsweise kann durch die Schlaganfalläsion das Zusammenspiel der Hemisphären gestört werden in Form einer gesteigerten Hemmung der ipsiläsionellen Hemisphäre durch Areale der intakten Hirnhälfte. Durch diese maladaptive Netzwerkstörung werden funktionelle Defizite, wie motorische Defizte, eine Aphasie oder ein Neglect, verstärkt [6, 7, 34-37].

Nicht-invasive Verfahren der Hirnstimulation wie TMS und transkranielle Gleichstromstimulation (tDCS) können kortikale Erregbarkeit sowie Neuroplastizität modulieren [38, 39]. Während für TMS diese Effekte im Zusammenhang mit den Plastizitätsphänomenen der Langzeitpotenzierung (LTP) beziehungsweise -depression (LTD), einer langandauernden Veränderung der synaptischen Übertagung von Nervenzellen, diskutiert werden [39, 40], werden Verschiebungen des Membranpotentials sowie deren Beeinflussung der neuronalen Entladungsrate [41-43] im Falle der tDCS als ursächlich angesehen. Es muss ferner betont werden, dass TMSoder tDCS-induzierte Veränderungen der regionalen Aktivität sich nachweislich auch auf axonal verbundene, aber räumlich entfernte Hirnregionen ausbreiten und dadurch die Aktivität innerhalb des gesamten Netzwerks beeinflussen können [44-48]. Beide Verfahren induzieren zudem Nacheffekte, welche die Zeit der reinen Stimulation überdauern $[39,49]$. In der Konsequenz scheinen beide Ansätze vielversprechend, um maladaptive Prozesse im Sinne der Netzwerkstörung nach einem Schlaganfall zu korrigieren und adaptive Reorganisationsvorgänge in der Rehabilitationsphase zu unterstützen. Dabei haben die oben vorgestellten Modelle der Interaktion der Hemisphären maßgeblich die Konzepte der therapeutischen Anwendung non-invasiver Hirnstimulation geformt. Analog zielte für jede der drei neurologischen Funktionsstörungen Parese, Aphasie und Neglect, auf welche sich die vorliegende Übersichtsarbeit konzentriert, die Intervention vornehmlich auf die Steigerung der Erregbarkeit der ipsiläsionellen Hemisphäre oder auf die Reduktion der Exzitabilität der kontraläsionellen Hemisphäre ab ( Abb. 1).

In Abhängigkeit der Stimulationsparameter können verschiedene Nacheffekte auf die kortikale Erregbarkeit erzielt werden. Im Allgemeinen gilt, dass repetitive TMS in Pulsfrequenzen $\leq 1 \mathrm{~Hz}$ sowie eine kathodale Stimulation der tDCS die kortikale Erregbarkeit reduzieren, während Stimulationsfrequenzen $\geq 5 \mathrm{~Hz}$ beziehungsweise eine anodale Stimulation die Erregbarkeit steigern [40, 50]. Für TMS haben sich darüber hinaus auch repetitive Protokolle, bei welchen die Pulse in spezifischen Mustern wie Theta-Burst-Stimulation (TBS) appliziert werden, etabliert [40]. Dabei sind kontinuierliche Pulsfolgen (cTBS) mit einer Abnahme und intermittierendes, also von Pausen unterbrochenes, TBS (iTBS) mit einer Steigerung der kortikalen Erregbarkeit vergesellschaftet [40]. Diese simplifizierten Prinzipien unterliegen bereits im Gesunden einem hohen Maß an interindividueller Variabilität und können durch zahlreiche Einflüsse wie neuroanatomische Aspekte, genetische Polymorphismen, die Grundaktivität des neuronalen Netzwerkes, aber auch technische Größen wie Stimulationsintensität sogar umgekehrt werden [51-55]. Die dem Schlaganfall inherente Heterogenität verstärkt aufgrund von Faktoren wie Zeit nach Schlaganfall, Läsionslokalisation und -größe, Komedikation oder Ausprägung des Defizits die Variabilität der Stimu- 


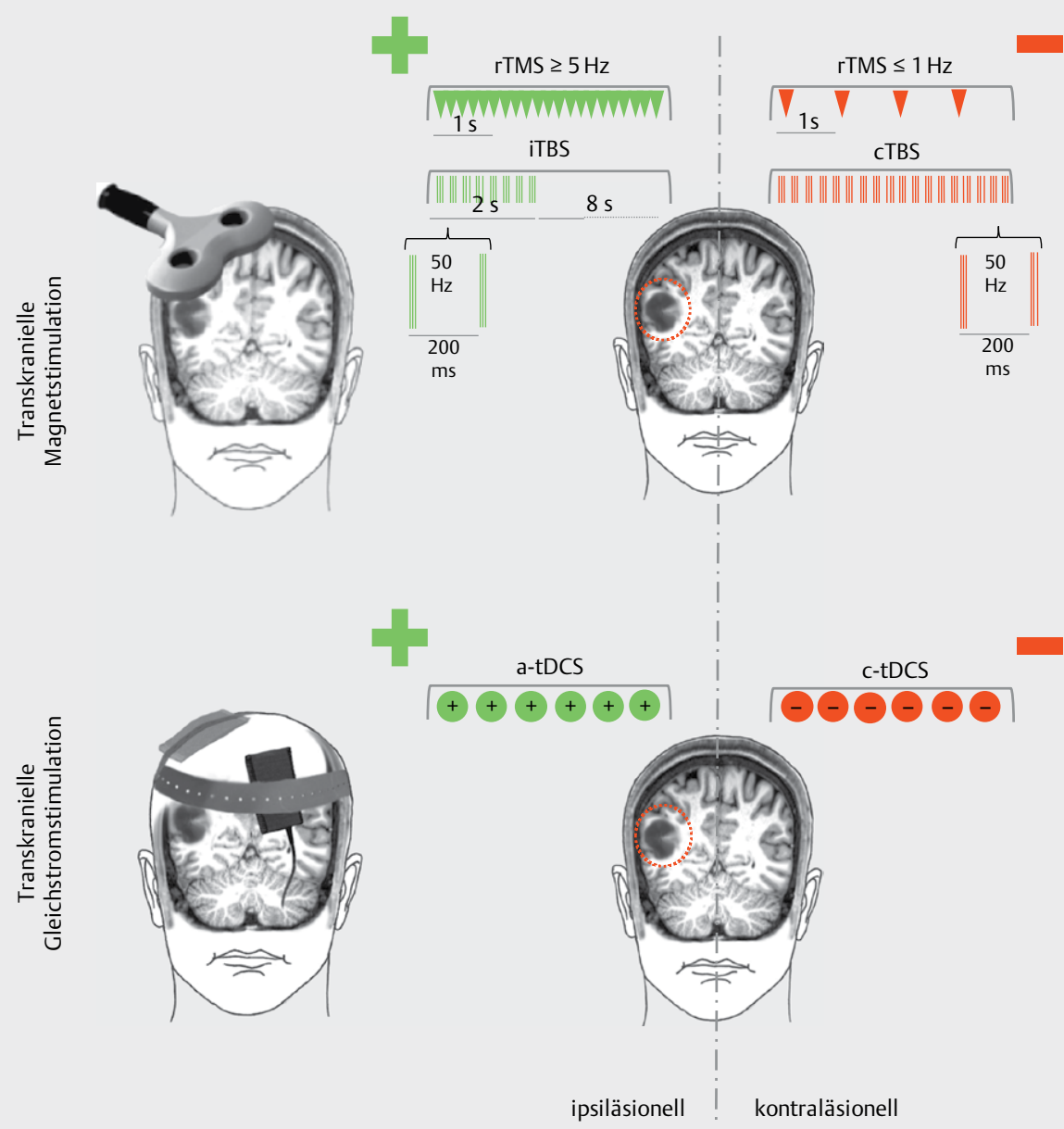

- Abb. 1 Nicht-invasive neuromodulatorische Techniken. Transkranielle Magnetstimulation (TMS): Die transkranielle Magnetstimulation beruht auf dem Prinzip der Faraday'schen elektromagnetischen Induktion. Dabei wird über eine Kupferspule, welche an die Schädeldecke gehalten wird, ein Magnetfeld im Mikrosekundebereich generiert, welches Haut und Knochen ungehindert passiert und im Hirngewebe ein elektrisches Feld induziert. Dadurch können Neurone und deren Axone unterhalb der TMS-Spule erregt werden. Im therapeutischen Einsatz wird die TMS repetitiv in mehreren hundert bis zu tausend aufeinanderfolgenden Pulsen angewandt. Abhängig von der Stimulationsfrequenz und Pulsfolge kann die Erregbarkeit erhöht ( $\geq 5 \mathrm{~Hz}$ rTMS) oder verringert ( $\leq 1 \mathrm{~Hz}$ rTMS) werden. TBS-Protokolle sind rTMS-Varianten, die je nach Muster der Stimulation (intermittierend vs. kontinuierlich) die kortikospinale Erregbarkeit entweder erhöhen (iTBS) oder verringern (cTBS) können. Bei allen rTMS Protokollen herrscht eine große inter-individuelle Variabilität hinsichtlich der induzierten Erregbarkeitsänderungen (gemessen mittels motorisch evozierter Potenziale). Transkranielle Gleichstromstimulation (tDCS): Bei der transkraniellen Gleichstromstimulation werden elektrische Ströme geringer Intensität (1-2 mA) kontinuierlich über Kopfhautelektroden über die Dauer von 10-20 Min appliziert. Im Gegensatz zur TMS, welche in Kombination mit einer Neuronavigation eine relativ fokale Stimulation darstellt, werden über die flächigen tDCS-Elektroden meist relativ große Teile des Gehirns erreicht. Die Modulation ist polaritätsspezifisch, das heißt, eine anodale Stimulation (a-tDCS) erhöht die Erregbarkeit, während eine kathodale Stimulation (c-tDCS) diese reduziert. Wie bei der rTMS unterliegen auch die tDCS-Effekte einer ausgeprägten inter-individuellen Variabilität.

lationseffekte vermutlich noch einmal um ein Vielfaches [56]. Zusammen mit der in der Literatur vorherrschenden Heterogenität der Anwendung non-invasiver Hirnstimulation in Bezug auf Stimulationsparameter, Anzahl der durchgeführten Stimulationen sowie Zeitpunkt und Art der begleitenden Therapie trägt es einerseits zu Fülle und Komplexität der Literatur bei und bedingt andererseits inkonsistente Studienergebnisse.

\section{Motorische Defizite}

Seit dem ersten Einsatz der rTMS zu therapeutischen Zwecken im Rahmen der Rehabilitation nach Schlaganfall vor nun fast 20 Jah- ren haben eine Reihe von Studien gezeigt, dass bereits nach einmaliger Stimulation die motorische Performanz von Schlaganfallpatienten mit Hemiparese verbessert werden kann [57-59]. Studien, welche in den letzten Jahren veröffentlicht wurden, haben dagegen vornehmlich versucht, die Effektivität der Intervention durch die Kombination mit einem nachfolgenden motorischen Training zu steigern und/oder durch serielle Stimulationen die Therapiedosis zu erhöhen [58]. Wie zuvor erwähnt, liegt den meisten Studien in der Konzeption der Stimulationsprotokolle das oben eingeführte Modell der interhemisphärischen Kompetition zu Grunde. Daraus resultiert, dass erregbarkeitssteigernde Protokolle (hochfrequente rTMS/iTBS, anodale tDCS) auf der läsionierten He- 
misphäre und erregbarkeitshemmende Protokolle (niedrigfrequente rTMS/cTBS, kathodale tDCS) auf der kontraläsionellen Hemisphäre eingesetzt werden sollten. Darüber hinaus bietet die tDCS durch eine entsprechende Montage der Elektroden auch die Möglichkeit einer bihemisphärischen Stimulation mit Platzierung der Anode über dem ipsiläsionellen Motorkortex und der Kathode über dem kontraläsionellen Motorkortex.

\section{rTMS}

Für die subakute Phase nach Schlaganfall konnte wiederholt ein Vorteil einer hochfrequenten rTMS beziehungsweise iTBS über dem ipsiläsionellen primär motorischen Kortex (M1) aufgezeigt werden [60-64]. So konnten wir bspw. zeigen, dass die tägliche Applikation von iTBS (600 Pulse, $70 \%$ der individuellen Ruhemotorschwelle (RMT)) innerhalb der ersten 2 Wochen nach einem Schlaganfall an fünf aufeinanderfolgenden Tagen vor der Physiotherapie zu einer stärkeren Verbesserungen der Griffkraft der paretischen Hand im Vergleich zur Kontrollgruppe führte [60]. Dieser Unterschied war auch bei einer Verlaufsmessung 3-6 Monate später noch nachweisbar. Die zusätzlich erhobenen fMRT-Daten ergaben, dass die Verum-Stimulationsgruppe nach der Interventionsphase im Vergleich zur Kontrollgruppe eine höhere intra- als interhemisphärische Konnektivität des ipsiläsionellen M1 aufwiesen [60]. In einer longitudinalen Studie von Guan und Kollegen konnte für eine hochfrequente rTMS über dem ipsiläsionellen M1 über zehn Tage sogar eine anhaltende motorische Verbesserung der oberen Extremität bis zu einem Jahr nach Schlaganfall gezeigt werden [61].

Auch der zweite Ansatzpunkt, die Reduktion der kontraläsionellen M1-Aktivität mittels inhibitorischer rTMS, kann in der subakuten Phase nach Schlaganfall zu einer Verbesserung der Motorik führen [63-67]. So konnten wir bspw. in früheren Studien bei Patienten in der späten Subakutphase, $2 \pm 1$ Monate nach Schlaganfall, zeigen, dass 1 Hz-rTMS (600 Pulse, 100\% RMT) nicht nur die motorische Performanz der paretischen Hand verbesserte, sondern dass diese Verbesserung sowohl mit einer Reduktion der Überaktivität in den stimulierten sowie ipsiläsionellen Regionen als auch mit einer Reduktion der inhibitorischen Einflüsse des kontraläsionellen M1 assoziiert war [69, 70].

Zwei weitere Studien verdeutlichen den kritischen Einfluss der Läsionslokalisation auf die Effektivität der Neuromodulation. Ameli und Kollegen fanden heraus, dass nur Patienten mit subkortikalen Läsionen auf eine ipsiläsionelle $10 \mathrm{~Hz}$ rTMS mit einer verbesserten motorischen Leistung sowie einer Normalisierung des fMRT-Aktivitätsmusters reagierten. Im Gegensatz dazu hatte dieses $10 \mathrm{~Hz}$ Protokoll über dem ipsiläsionellen $\mathrm{M} 1$ bei Patienten mit kortikalen Infarkten weder einen positiven Einfluss auf die motorische Performanz noch auf die Hirnaktivität [68]. Lüdemann-Podubecká und Kollegen fanden weiteren Anhalt, dass die Wirkung einer kontraläsionellen $1 \mathrm{~Hz}$-Stimulation (900 Pulse, 100\% RMT) auch davon abhängen kann, ob die dominante oder nicht-dominante Hemisphäre vom Schlaganfall betroffen ist. Die Autoren identifizierten hier nur positive rTMS-vermittelte Verhaltenseffekte, wenn der Motorkortex der dominanten Hemisphäre mittels niederfrequenter Stimulation beeinflusst wurde [66].

Erste Hinweise legen nahe, dass die Kombination beider Protokolle, also eine kontraläsionelle, niedrigfrequente rTMS $(1 \mathrm{~Hz}, 1000$ Pulse, $90 \%$ RMT) gefolgt von einer ipsiläsionellen, erregbarkeits- steigernden Stimulation (10 Hz, 1000 Pulse, 90\% RMT), sogar noch wirksamer zu sein scheint [69].

Im chronischen Stadium nach Schlaganfall konnte kompatibel mit dem Konzept der interhemisphärischen Kompetition konsistent über Studien hinweg eine erhöhte Aktivität des kontraläsionellen M1 sowie eine gesteigerte interhemisphärische Inhibition von diesem auf die geschädigte Hemisphäre gezeigt werden $[34,70]$. Erste Studien in kleinen Patientenkollektiven bestätigten auch die theoretischen Überlegungen einer Reduktion der kontraläsionell gesteigerten kortikale Erregbarkeit mittels niedrigfrequenter rTMS [71-75]. Allerdings konnten diese Ergebnisse 2018 in einer hochwertigen, multizentrischen Studie mit hoher Patientenanzahl (199 Patienten mittelgradiges residuelles motorisches Defizit, 3-12 Monate nach Schlaganfall) nicht bestätigt werden [76]: Im NICHE Trial erfolgten über sechs Wochen insgesamt 18 Sitzungen $1 \mathrm{~Hz}$-rTMS (900 Pulse, elektrisches Feld: 110 V/m) gefolgt von einem 60-minütigen Armtraining [76]. Am Endpunkt nach 6 Monaten wiesen sowohl die Verumstimulationsgruppe als auch die Kontrollgruppe eine Verbesserung aller motorischen Parameter gegenüber der Baseline auf. Ein zusätzlicher Vorteil der $1 \mathrm{~Hz}$-Stimulation konnte jedoch nicht gefunden werden, sodass resümiert werden muss, dass zumindest für Patienten im frühen chronischen Stadium mit moderatem motorischem Defizit eine $1 \mathrm{~Hz}-\mathrm{rTMS}$ den Effekt einer motorischen Therapie nicht zusätzlich verbessern kann. Darüber hinaus verdeutlicht dieses Beispiel, dass auch wenn die Ergebnisse von Machbarkeitsstudien mit geringen Patientenzahlen im Einklang mit mechanistischen Theorien stehen und wichtige Informationen über das Potenzial von neuromodulatorischen Therapie liefern können, am Ende jedoch ausreichend gepowerte Studien den Wert eines Interventionsprotokolls in der klinischen Praxis bestimmen.

Beispiele für die Förderung der Rehabilitation der motorischen Handfunktion anhand von ipsiläsioneller, hochfrequenter rTMS in der chronischen Phase nach Schlaganfall verdeutlichen die kritische Rolle, welcher der Zeit nach Schlaganfall wohl für die Effektivität der Neuromodulation innewohnt. So hatte eine Sitzung iTBS bei Patienten mit chronischen motorischen Defiziten ( $>12$ Monate nach Schlaganfall) auf Gruppenebene keinen Verhaltenseffekt, obwohl die Erregbarkeit des ipsiläsionellen M1 zunahm [77].

Insgesamt gesehen ist somit die Datenlage für den Einsatz der rTMS zur motorischen Rehabilitation als gemischt anzusehen [78]. Daher sollte der therapeutische Einsatz dieser Methode stets wissenschaftlich begleitet sein, um systematisch diejenigen Patienten identifizieren zu können, welche davon profitieren.

\section{tDCS}

Während im subakuten Stadium Proof-of-Principle Studien an kleinen Patientenstichproben immer wieder einen Nutzen der tDCS für die Rehabilitation motorischer Defizite andeuten [79-81], haben 2 große klinische Studien keinen Vorteil finden können $[82,83]$. Die erste randomisierte, doppelverblindete, multizentrische Studie an 96 Patienten in der subakuten Phase nach Schlaganfall zeigte sowohl für eine ipsiläsionelle, anodale Stimulation als auch für eine kontraläsionelle, kathodale Stimulation jeweils gefolgt von einem roboterassistierten Armtraining über einen Zeitraum von 6 Wochen (30 Sitzungen, 2 mA über 20 Min) keinen zusätzlichen Nutzen gegenüber einer Kontrollstimulation [82]. Rossi 
und Kollegen konnten für eine ipsiläsionelle, anodale tDCS über 5 konsekutive Tage ( $2 \mathrm{~mA}$ über 20 Minuten) in einer Gruppe von 50 akuten Schlaganfallpatienten (2 Tage nach Ereignis) ebenfalls keinen Überlegenheitsvorteil der tDCS für die Erholung der motorischen Funktion zeigen [83].

In der chronischen Phase zeigte die Gruppe um Johansen-Berg, dass eine anodale Stimulation des ipsiläsionellen M1 (9 Sitzungen, $1 \mathrm{~mA}$ über $20 \mathrm{Min}$ ) gefolgt von motorischem Training an 9 aufeinanderfolgenden Tagen nicht nur die motorische Performanz von chronischen Schlaganfallpatienten erhöht, sondern, auch mit einer erhöhten Aktivität des ipsiläsionellen prä- und primär motorischen Kortex einhergeht [84]. Insgesamt sind jedoch die Ergebnisse von tDCS-Studien in der chronischen Phase sehr heterogen mit teils fehlenden Stimulationseffekt [84-86]. In Zusammenschau muss zum aktuellen Zeitpunkt konstatiert werden, dass es für motorische Defizite sowohl in der subakuten als auch chronischen Phase nach Schlaganfall für keines der aktuell zur Anwendung kommenden Protokolle hinreichende Evidenzen für einen konsistent messbaren Nutzen der tDCS gibt [87].

\section{Aphasie}

Analog der kortikalen Veränderungen und Reorganisationsprozesse, welche im Rahmen schlaganfallbedingter Störungen der Willkürmotorik aufgedeckt werden konnten, scheinen auch Sprachstörungen mit einer erhöhten Aktivität in anatomisch homologen Regionen der rechten, kontraläsionellen Hemisphäre während entsprechender Aufgaben einherzugehen [6,88]. Diese Überaktivität wurde gleichfalls im Rahmen von maladaptiver Plastizität und fehlender interhemisphärischen Inhibition innerhalb des neuronalen Netzwerks gesehen [30]. Dementsprechend ist es gleichsam das Ziel der Behandlung aphasischer Defizite nach einem Schlaganfall, die ipsiläsionelle Exzitabilität zu steigern, die Erregbarkeit maladaptiv überaktiver kontraläsioneller Areale zu reduzieren oder im Falle von tDCS eine Kombination beider Verfahren anzuwenden. Da sich eine intensive Sprachtherapie als eine der wirksamsten Behandlungen zur Verbesserung der Aphasie bei Schlaganfallpatienten etabliert hat [89], sind neuromodulatorische Verfahren, analog motorischer Störungen, ebenfalls zumeist kombiniert mit Sprachtherapie.

\section{rTMS}

Die Literatur hinsichtlich des Einsatzes von rTMS zur Augmentation der Rehabilitation aphasischer Defizite in der subakuten Phase nach Schlaganfall ist limitiert, wenn auch wenige Studien erste Hinweise für einen zusätzlichen Nutzen einer niederfrequenten rTMS über dem rechten, kontraläsionellen Gyrus frontalis inferior (IFG), erbracht haben [90-93]. Eine neuere Studie konnte an einer Gruppe von 30 Schlaganfallpatienten mit gemischt aphasischen Störungen in der Subakutphase zeigen, dass eine $1 \mathrm{~Hz}$ rTMS (1200 Pulse, 90 \% RMT) über dem rechten IFG unmittelbar vor einem 45-minütigen logopädischen Training gegenüber der Kontrollgruppe eine verbesserte Sprachleistung nach 10-tägige Intervention bewirkt [94].

Im chronischen Stadium nach Schlaganfall fokussieren sich die in den letzten Jahren publizierten Studien v. a. auf die rTMS Behandlung von Patienten mit expressiven Aphasien [95-98]. Während eine Studie zeigen konnten, dass ein 10-tägiges 1Hz-rTMS-Protokoll (600 Pulse, $80 \%$ RMT) über dem rechten IFG zu einer Verbesserung der Aphasie gegenüber einer gleichartig applizierten $10 \mathrm{~Hz}$ Stimulation führen kann und diese Effekte auch noch nach 2 Monaten nachweisbar sind [96], demonstrierten 2 weitere Studien, dass $1 \mathrm{~Hz}-$-rTMS (600 bzw. 1200 Pulse, $90 \%$ RMT) über der Pars triangularis des IFG zu einer verbesserten Objekt- und Handlungsbenennung führt, welche sich auch noch drei Monaten nach der Intervention nachvollziehen lässt $[97,98]$.

\section{tDCS}

In der subakuten Phase nach einem Schlaganfall kann die Datenlage einen nachhaltigen Effekt der tDCS auf die Rehabilitation aphasischer Störungen bislang nicht überzeugend unterstützen [99]. Neben Hinweisen aus Einzelfallberichten und Studien an kleinen Patientenstichproben ( $\leq 10$ Patienten) $[100,101]$ deuten erste Ergebnisse von You und Kollegen an 21 subakuten Schlaganfallpatienten mit expressiv-betonter Aphasie auf einen potentiellen Nutzen einer kathodalen Stimulation über dem rechten Gyrus temporalis superior für die Erholung des Sprachverständnisses hin [102].

Eine erst rezent publizierte, randomisierte und doppelverblindete Multizenterstudie, die fast 60 Schlaganfallpatienten in der subakuten Phase einschloss, zeigte hingegen für die Kombination von anodaler tDCS (10 Sitzungen, $1 \mathrm{~mA}$ über $20 \mathrm{~min}$ ) über dem linken IFG und nachfolgendem 45-minütigen Sprachtraining keinerlei Überlegenheitsvorteil gegenüber der Kontrollstimulation [103] und relativiert somit die Hinweise eines möglichen Nutzen einer anodalen, ipsiläsionellen tDCS im subakuten Stadium, welcher sich aus einer 2013 publizierten Studie ergab [104].

In der chronischen Phase hingegen bestätigte zuletzt eine randomisierte, doppelverblindete klinische Studie [105] die Ergebnisse mehrerer vorheriger Machbarkeitsstudien [106-109]. Bei 74 chronischen Schlaganfallpatienten führte eine anodale tDCS (15 Sitzungen, $1 \mathrm{~mA}$ über $20 \mathrm{~min}$ ) über der linken Temporalregion zusätzlich zu einem computergesteuerten Sprachtraining über 3 Wochen zu einer größeren Verbesserung in der Benennung von Objekten als eine Scheinstimulation [105]. Die Ergebnisse dieser Arbeit lassen weitere Bemühungen hinsichtlich der tDCS zur Verbesserung der Rehabilitation aphasischer Störungen nach Schlaganfall gerechtfertigt erscheinen. Dementsprechend kommt auch eine Cochrane Metaanalyse zu dem Schluss, dass obwohl die Datenlage hinsichtlich einer Verbesserung einer alltagsrelevanten funktionellen Kommunikation nicht ausreichend ist, es sowohl direkt nach Intervention als auch für eine Follow-Up-Periode Hinweise für einen Effekt der tDCS zur Verbesserung der Benenngenauigkeit gibt [99].

\section{Neglect}

Auch bei der Behandlung von räumlichen Aufmerksamkeitsstörungen gründet der Ansatzpunkt auf dem Modell der interhemisphärischen Kompetition und einer Erregbarkeitssteigerung des ipsiläsionellen Parietalkortex oder Erregbarkeitssenkung des kontraläsionellen Parietalkortex $[110,111]$. Charakteristischerweise tritt ein Neglect zumeist in Folge von Läsionen im Bereich des rechten posterioren parietalen Kortex (PPC) oder des hinteren Teils des Gyrus temporal superior (STG) auf [77] und betrifft somit bevor- 
zugt die linke Raum- und Körperseite. Dem Modell folgend führt eine entsprechende Läsion der rechten Hemisphäre zu einer reduzierten transkallosalen Inhibition der kontraläsionellen intakten homologen Region und zu einer pathologisch erhöhten Aktivität, welche die visuell-räumliche Aufmerksamkeit der ipsiläsionellen Seite des Raumes verzerrt und so zu einer räumlichen Vernachlässigung führt $[7,36,112]$.

\section{rTMS}

Insgesamt ist die Anzahl von Studien zur rTMS-Therapie des Neglects deutlich geringer als für die zuvor diskutierten Defizite. Hier stammen bestätigende Hinweise für einen Nutzen der rTMS vor allem aus Studien, in welchen das inhibierende Protokoll der cTBS zum Einsatz kam [112-116]. So zeigten Koch und Kollegen, dass bei einer Gruppe von 20 Schlaganfallpatienten im subakuten Stadium eine cTBS (600 Pulse, 80\% AMT) über dem linken kontraläsionellen PPC für 10 Behandlungstage zu einer Verbesserung des Neglects gegenüber der Kontrollstimulation führte, und die Nacheffekte auch 2 Wochen nach der Behandlung andauerten [113]. Diese Ergebnisse konnten in einer weiteren Studie für ein ähnliches cTBS-Protokoll ( $30 \mathrm{~Hz}$ Bursts) erneut bestätigt werden [116]. Hier war die Verbesserung durch die Stimulation auch noch im Beobachtungsintervall von 4 Wochen nachvollziehbar. In diesem Zusammenhang erwähnenswert sind ebenfalls die Arbeiten von Nyffeler und Kollegen. Einerseits stehen ihre Ergebnisse für die cTBS des linken PPC in Kongruenz zu den oben genannten Studien [112, 114, 115]. Auf der anderen Seite gelang es ihnen kürzlich, einen Teil der Variabilität der Reaktion auf cTBS durch die Integrität von interhemisphärischen parieto-parietalen Verbindungen innerhalb des Corpus callosum aufzuklären [114] und so einen wichtigen Beitrag zu der Diskussion über die Entwicklung und Optimierung effektiver Stimulationsprotokolle auf individueller Basis zu liefern.

\section{tDCS}

Wenn auch die Literatur hinsichtlich der Unterstützung der Rehabilitation eines schlaganfallbedingten Neglects mittels tDCS limitiert ist und zumeist auf kleinen Patientengruppen oder Fallberichten basiert [117-120], gibt es dennoch Hinweise aus 2 Studien, welche an einer Stichprobe von jeweils 30 Schlaganfallpatienten einen Nutzen der tDCS nahe legen $[110,111]$. Ihnen gemein ist, dass sie sowohl die Effekte einer anodalen, ipsiläsionell als auch einer kathodalen, kontraläsionellen Stimulation gegenüber einer Scheinstimulation untersucht haben. Dabei wurden in 2- (10 Sitzungen, $2 \mathrm{~mA}$ über $30 \mathrm{~min}$ ) [111] beziehungsweise 3-wöchigen (15 Sitzungen, 2 mA über 30 min) [110] Interventionen die tDCS mit Therapieverfahren zur Behandlung des Neglects kombiniert. Hier zeigte sich insbesondere ein übereinstimmender Stimulationseffekt für die anodale tDCS, wohingegen eine kathodale Stimulation widersprüchliche Ergebnisse erzielte. Somit gilt auch für dieses Verfahren, dass größere, ausreichend gepowerte Studien notwendig sind, um den Wert der tDCS in der Behandlung des Neglects einordnen zu können.

\section{Zusammenfassung}

Die hier vorgestellten Studien zur nicht-invasiven Hirnstimulation liefern in ihrem Grundkonzept vielversprechende Ansätze zur Un- terstützung der Funktionserholung nach einem Schlaganfall. Auch wenn Kliniken und Praxen dieses Verfahren vereinzelt bereits therapeutisch einsetzen, hat die nicht-invasive Neurostimulation noch keinen Einzug in die routinemäßige Rehabilitationstherapie gehalten. Unklar ist auch, wie sehr sich die stimulationsvermittelten Verbesserungen einzelner Parameter wie Griffkraft auf die Alltagskompetenzen der Patienten übertragen.

Die Gründe für die bisher sehr geringe klinische Translation liegen einerseits in dem Umstand, dass das Forschungsfeld von einer Vielzahl von Studien mit geringer Stichprobengröße beherrscht wird, wohingegen groß angelegte, randomisierte Multicenterstudien eher rar sind $[76,105]$. Der metaanalytische Vergleich der Wirksamkeit der Verfahren wird auch erschwert durch die ausgeprägte Heterogenität der zur Anwendung kommenden Stimulationsprotokolle (Intensität, Anzahl der durchgeführten Sitzungen), des Zeitpunkt und der Art der begleitenden physikalischen Therapie sowie der Outcome-Parameter, welche den Erfolg des Behandlungsverfahrens messen sollen, gekennzeichnet sind. Daher erscheinen ausreichend gepowerte Studien mit großen (in der Regel > 100 Probanden) Stichproben notwendig, welche basierend auf standardisierten Stimulations-, Behandlungs- und Outcome-Parametern durchgeführt werden. In diesem Sinne ist bereits eine positive Entwicklung zu vernehmen und sowohl für rTMS als auch tDCS eine kleine Anzahl registrierter klinischer Studien zu verzeichnen (clinicalTrials.gov: Theta-Burst-Stimulation in Early Rehabilitation of Stroke (TheSiReS), NCT02910024 [131]; Neuroregeneration Enhanced by Transcranial Direct Current Stimulation (TDCS) in Stroke (NETS), NCT00909714; Rehabilitating (Stroke-induced) Apraxia With Direct Current Stimulation (RAdiCS), NCT03185234 [132]).

Neben der Heterogenität der Neuromodulationsstudien weisen aber Effekte non-invasiver Hirnstimulation bereits in gesunden Probanden ein erhebliches Maß an interindividuelle Variabilität hinsichtlich der induzierten Änderung der kortikalen Erregbarkeit auf [51]. Abgesehen davon, dass die Änderung der kortikalen Erregbarkeit zumeist als Änderung der relativ störanfällige Messgröße der Amplituden von motorisch evozierten Potenzialen (MEPs) gemessen wird und somit die Interventionseffekte auf neuronaler Ebene nur sehr begrenzt erfasst, besteht eine zusätzliche Herausforderung in der Variabilität, die das Kollektiv der Schlaganfallpatienten in Hinblick auf Alter, Vorschädigungen des Gehirns, individuelle Betroffenheit, Zeit nach Schlaganfall sowie Läsionsgröße und -lokalisation mit sich bringt. Aktuelle Stimulationsregimes folgen in der Regel einer einheitlichen Strategie für alle Patienten. Das Konzept der interhemisphärischen Kompetition, welches als grundlegende Rationale vieler neuromodulativer Studien gilt, scheint jedoch viel zu simplifiziert, um individuell in jedem Patienten erfolgreich angewendet werden zu können. Funktionelle Bildgebungsstudien haben gezeigt, dass sich die Reorganisationsmuster und die Störung der interhemisphärischen Konnektivität teilweise erheblich zwischen Patienten unterscheiden. Dazu passend fallen auch die Stimulationseffekte individuell sehr unterschiedlich aus. Beispielsweise konnten wir zeigen, dass sich die Griffkraft unter Physiotherapie kombiniert mit iTBS des ipsiläsionellen M1 zwar im Mittel um 21 Prozentpunkte gegenüber 11 Prozentpunkte bei der Kontrollstimulation verbesserte, die Spannbreite in der Verumgruppe jedoch von 0 bis $44 \%$ Verbesserung betrug (Placebogruppe: 0-25\%) [60]. Somit gibt es ein erhebliches Maß an individueller Variabilität hinsichtlich des Ansprechens auf 
eine therapeutische Hirnstimulation, was vor dem Hintergrund der individuell sehr unterschiedlichen Reorganisationsmuster nicht sehr verwundert. Daher erscheint eine an der individuellen Netzwerkpathologie orientierte Stimulationsstrategie eher geeignet, die Reorganisation gestörter Netzwerke zu unterstützen.

Auf dem Weg zu personalisierten Therapieansätzen sollten auch Stimulationsorte außerhalb der konventionellen Zielorte diskutiert werden. Beispielsweise spielen bei der motorischen Rehabilitation neben M1 auch übergeordnete sensomotorische Areale, wie der ventrale und dorsale prämotorische Kortex, der supplementär motorische Kortex oder der posteriore Parietalkortex, eine wichtige Rolle [5, 57]. Ihre Relevanz für die Durchführung von Bewegungen der paretischen Hand konnte in online-TMS-Studien bestätigt werden [121-123]. Eine weitere Voraussetzung für eine individualisierte Therapie ist eine personalisierte, multimodale Prädiagnostik. Doch auch wenn die funktionelle Bildgebung das Verständnis der neuronalen Mechanismen der Funktionserholung nach Schlaganfall und der durch die Hirnstimulation hervorgerufenen Veränderungen maßgeblich verbessert hat, sind wir noch weit von einem personalisierten Ansatz entfernt. Daher sind methodische Weiterentwicklungen gefordert, welche nicht nur mit ausreichender Zuverlässigkeit und Validität die individuelle Netzwerkpathologie eines Patienten erfassen, sondern auch auf individueller Ebene das Ansprechen auf eine neuromodulative Therapie widerspiegeln können. Zwar wurden bereits erste Versuche unternommen, mittels multivariater maschineller Lernverfahren das motorische Defizit oder motorische Outcome in Patienten auf der Grundlage von fMRTDaten zu prädizieren $[124,125]$. Jedoch beobachteten Siegel und Kollegen, dass nur etwa $20 \%$ der beobachteten Varianz des motorischen Defizits durch funktionelle Konnektivität erklärt werden können [126]. Daher bleibt es Gegenstand zukünftiger Forschung, welche Methodik oder welche multimodale Kombination ausreichend genaue Surrogatmarker für eine individuelle Patientencharakterisierung liefern kann. Denkbar wäre, dass das EEG hier wichtige Informationen liefert, da dieses Verfahren sowohl die Aktivität als auch Konnektivität des Gehirns darstellen und somit unmittelbar über die funktionelle Integrität des kortikalen Gewebes informieren kann [31, 130]. In Kombination mit TMS ergibt sich die Möglichkeit, die Echtzeitinformationen des EEGs zu nutzen, um die TMS im Sinne eines closed-loop-Verfahrens kontrolliert zu applizieren [127]. Erste Ergebnisse in gesunden Probanden legen nahe, dass durch die Information über den instantanen Zustand des Gehirns Stimulationseffekte augmentiert und die Wirksamkeit der Plastizitätsinduktion gesteuert werden kann [128]. Während die Machbarkeit nun auch kürzlich in Schlaganfallpatienten berichtet worden ist, bleibt ihr tatsächlicher Nutzen in zukünftigen Studien zu evaluieren [129].

Interessenkonflikt

Die Autorinnen/Autoren geben an, dass kein Interessenkonflikt besteht.
Literatur

[1] Carmichael ST. Cellular and molecular mechanisms of neural repair after stroke: Making waves. Ann Neurol 2006; 59: 735-742

[2] Cramer SC. Repairing the human brain after stroke: I. Mechanisms of spontaneous recovery. Ann Neurol 2008; 63: 272-287

[3] Lakhan SE, Kirchgessner A, Hofer M. Inflammatory mechanisms in ischemic stroke: therapeutic approaches. J Transl Med 2009; 7: 97

[4] Nudo RJ. Recovery after brain injury: mechanisms and principles. Front Hum Neurosci 2013; 7: 887

[5] Rehme AK, Fink GR, Cramon von DY, u. a. The role of the contralesional motor cortex for motor recovery in the early days after stroke assessed with longitudinal FMRI. Cereb Cortex 2011; 21: 756-768

[6] Heiss WD, Thiel A. A proposed regional hierarchy in recovery of post-stroke aphasia. Brain and Language 2006; 98: 118-123

[7] Corbetta M, Kincade M], Lewis C, u. a. Neural basis and recovery of spatial attention deficits in spatial neglect. Nat Neurosci 2005; 8: $1603-1610$

[8] Stockert A, Wawrzyniak M, Klingbeil ], u. a. Dynamics of language reorganization after left temporo-parietal and frontal stroke. Brain 2020; 143: 844-861

[9] Langhorne P, Bernhardt J, Kwakkel G. Stroke rehabilitation. The Lancet 2011; 377: 1693-1702

[10] Kwakkel G, Kollen B, Lindeman E. Understanding the pattern of functional recovery after stroke: facts and theories. Restorative Neurology and Neuroscience 2004; 22: 281-299

[11] Zeiler SR, Krakauer JW. The interaction between training and plasticity in the poststroke brain. Current Opinion in Neurology 2013; 26: 609-616

[12] Bernhardt J, Hayward KS, Kwakkel G, u. a. Agreed Definitions and a Shared Vision for New Standards in Stroke Recovery Research: The Stroke Recovery and Rehabilitation Roundtable Taskforce. Neurorehabilitation and Neural Repair 2017; 31: 793-799

[13] Prabhakaran S, Zarahn E, Riley C, u. a. Inter-individual Variability in the Capacity for Motor Recovery After Ischemic Stroke. Neurorehabilitation and Neural Repair 2007; 22: 64-71

[14] Vliet R, Selles RW, Andrinopoulou ER, u. a. Predicting upper limb motor impairment recovery after stroke: a mixture model. Ann Neurol 2020; ana. 25679-25623

[15] Nijboer TCW, Kollen BJ, Kwakkel G. Time course of visuospatial neglect early after stroke: a longitudinal cohort study. Cortex 2013; 49: 2021-2027

[16] Lazar RM, Speizer AE, Festa JR, u. a. Variability in language recovery after first-time stroke. J Neurol Neurosurg Psychiatr 2008; 79 : 530-534

[17] Dobkin BH, Carmichael ST. The Specific Requirements of Neural Repair Trials for Stroke. Neurorehabilitation and Neural Repair 2015; 30: $470-478$

[18] Krakauer JW, Carmichael ST, Corbett D, u. a. Getting Neurorehabilitation Right. Neurorehabilitation and Neural Repair 2012; 26: 923-931

[19] Biernaskie J. Efficacy of Rehabilitative Experience Declines with Time after Focal Ischemic Brain Injury. J Neurosci 2004; 24: 1245-1254

[20] Clarkson AN, Overman J], Zhong S, u. a. AMPA Receptor-Induced Local Brain-Derived Neurotrophic Factor Signaling Mediates Motor Recovery after Stroke. J Neurosci 2011; 31: 3766-3775

[21] Duncan PW, Goldstein LB, Matchar D, u. a. Measurement of motor recovery after stroke. Outcome assessment and sample size requirements. Stroke 1992; 23: 1084-1089

[22] Stinear CM, Barber PA, Petoe M, u. a. The PREP algorithm predicts potential for upper limb recovery after stroke. Brain 2012; 135: $2527-2535$ 
[23] Nakayama H, Jørgensen HS, Raaschou HO, u. a. The influence of age on stroke outcome. The Copenhagen Stroke Study. Stroke 1994; 25: 808-813

[24] Feng W, Wang J, Chhatbar PY, u. a. Corticospinal tract lesion load: An imaging biomarker for stroke motor outcomes. Ann Neurol 2015; 78: $860-870$

[25] Goldstein LB, Samsa GP, Matchar DB, u. a. Charlson Index Comorbidity Adjustment for Ischemic Stroke Outcome Studies. Stroke 2004; 35: 1941-1945

[26] Grefkes C, Fink GR. Connectivity-based approaches in stroke and recovery of function. The Lancet Neurology 2014; 13: 206-216

[27] Heiss WD, Kessler J, Thiel A, u. a. Differential capacity of left and right hemispheric areas for compensation of poststroke aphasia. Ann Neurol 1999; 45: 430-438

[28] Léger A, Démonet J-F, Ruff S, u. a. Neural Substrates of Spoken Language Rehabilitation in an Aphasic Patient: An fMRI Study. Neurolmage 2002; 17: 174-183

[29] Belin P, Van Eeckhout P, Zilbovicius M, u. a. Recovery from nonfluent aphasia after melodic intonation therapy: a PET study. Neurology 1996; 47: 1504-1511

[30] Rosen HJ, Petersen SE, Linenweber MR, u. a. Neural correlates of recovery from aphasia after damage to left inferior frontal cortex. Neurology 2000; 55: 1883-1894

[31] Auriat AM, Neva JL, Peters S, u. a. A Review of Transcranial Magnetic Stimulation and Multimodal Neuroimaging to Characterize Post-Stroke Neuroplasticity. Front Neurol 2015; 6: 226

[32] Xu J, Branscheidt M, Schambra H, u. a. Rethinking interhemispheric imbalance as a target for stroke neurorehabilitation. Ann Neurol 2019; $1-36$

[33] Friston K], Harrison L, Penny W. Dynamic causal modelling Neurolmage 2003; 19: 1273-1302

[34] Grefkes C, Nowak DA, Eickhoff SB, u. a. Cortical connectivity after subcortical stroke assessed with functional magnetic resonance imaging. Ann Neurol 2008; 63: 236-246

[35] Koch G, Oliveri M, Cheeran B, u. a. Hyperexcitability of parietal-motor functional connections in the intact left-hemisphere of patients with neglect. Brain 2008; 131: 3147-3155

[36] Kinsbourne M. Hemi-neglect and hemisphere rivalry. Adv Neurol 1977; 18: 41-49

[37] Geranmayeh F, Brownsett SLE, Wise RJS. Task-induced brain activity in aphasic stroke patients: what is driving recovery? Brain 2014; 137: 2632-2648

[38] Lefaucheur J-P, Aleman A, Baeken C, u. a. Evidence-based guidelines on the therapeutic use of repetitive transcranial magnetic stimulation (rTMS): An update (2014-2018). Clinical Neurophysiology 2020: 474-528

[39] Rossini PM, Burke D, Chen R, u. a. Non-invasive electrical and magnetic stimulation of the brain, spinal cord, roots and peripheral nerves: Basic principles and procedures for routine clinical and research application. An updated report from an I.F.C.N. Committee. Clin Neurophysiol 2015; 126: 1071-1107

[40] Huang Y-Z, Edwards M], Rounis E, u. a. Theta Burst Stimulation of the Human Motor Cortex. Neuron 2005; 45: 201-206

[41] Nitsche MA, Paulus W. Excitability changes induced in the human motor cortex by weak transcranial direct current stimulation. J Physiol (Lond) 2000; 527: 633-639

[42] Nitsche MA, Paulus W. Transcranial direct current stimulation - update 2011. Restorative Neurology and Neuroscience 2011; 29: 463-492

[43] Nitsche MA, Fricke K, Henschke U, u. a. Pharmacological modulation of cortical excitability shifts induced by transcranial direct current stimulation in humans. J Physiol (Lond) 2003; 553: 293-301
[44] Bestmann S, Baudewig ], Siebner HR, u. a. BOLD MRI responses to repetitive TMS over human dorsal premotor cortex. Neurolmage 2005;

[45] Nettekoven C, Volz LJ, Kutscha M, u. a. Dose-Dependent Effects of Theta Burst rTMS on Cortical Excitability and Resting-State Connectivity of the Human Motor System. Journal of Neuroscience 2014; 34: 6849-6859

[46] Polanía R, Paulus W, Nitsche MA. Modulating cortico-striatal and thalamo-cortical functional connectivity with transcranial direct current stimulation. Hum Brain Mapp 2011; 33: 2499-2508

[47] Polanía R, Nitsche MA, Paulus W. Modulating functional connectivity patterns and topological functional organization of the human brain with transcranial direct current stimulation. Hum Brain Mapp 2010; 32: 1236-1249

[48] Keeser D, Meindl T, Bor J, u. a. Prefrontal transcranial direct current stimulation changes connectivity of resting-state networks during fMRI. Journal of Neuroscience 2011; 31: 15284-15293

[49] Liew S-L, Santarnecchi E, Buch ER , u. a. Non-invasive brain stimulation in neurorehabilitation: local and distant effects for motor recovery. Front Hum Neurosci 2014; 8: 265

[50] Rothkegel H, Sommer M, Paulus W. Breaks during $5 \mathrm{~Hz}$ rTMS are essential for facilitatory after effects. Clin Neurophysiol 2010; 121: 426-430

[51] Hamada M, Murase N, Hasan A, u. a. The role of interneuron networks in driving human motor cortical plasticity. Cereb Cortex 2013; 23: 1593-1605

[52] Cheeran B, Talelli P, Mori F, u. a. A common polymorphism in the brain-derived neurotrophic factor gene (BDNF) modulates human cortical plasticity and the response to rTMS. The Journal of Physiology 2008; 586: 5717-5725

[53] Batsikadze G, Moliadze V, Paulus W, u. a. Partially non-linear stimulation intensity-dependent effects of direct current stimulation on motor cortex excitability in humans. The Journal of Physiology 2013: 591: 1987-2000

[54] Bienenstock EL, Cooper LN, Munro PW. Theory for the development of neuron selectivity: orientation specificity and binocular interaction in visual cortex. J Neurosci 1982; 2: 32-48

[55] Müller-Dahlhaus F, Ziemann U. Metaplasticity in Human Cortex. The Neuroscientist 2015; 21: 185-202

[56] Grefkes C, Ward NS. Cortical Reorganization After Stroke: How Much and How Functional? The Neuroscientist 2014; 20: 56-70

[57] Hummel FC, Celnik P, Pascual-Leone A, u. a. Controversy: Noninvasive and invasive cortical stimulation show efficacy in treating stroke patients. Brain Stimul 2008; 1: 370-382

[58] Lefaucheur J-P, André-Obadia N, Antal A, u. a. Evidence-based guidelines on the therapeutic use of repetitive transcranial magnetic stimulation (rTMS). Clinical Neurophysiology 2014; 125: 2150-2206

[59] Lefaucheur J-P, Antal A, Ayache SS, u. a. Evidence-based guidelines on the therapeutic use of transcranial direct current stimulation (tDCS). Clinical Neurophysiology 2017; 128: 56-92

[60] Volz LJ, Rehme AK, Michely J, u. a. Shaping Early Reorganization of Neural Networks Promotes Motor Function after Stroke. Cerebral Cortex 2016; bhw034

[61] Guan Y-Z, Li ], Zhang X-W, u. a. Effectiveness of repetitive transcranial magnetic stimulation (rTMS) after acute stroke: A one-year longitudinal randomized trial. CNS Neurosci Ther 2017; 23: 940-946

[62] Hosomi K, Morris S, Sakamoto T, u. a. Daily Repetitive Transcranial Magnetic Stimulation for Poststroke Upper Limb Paresis in the Subacute Period. J Stroke Cerebrovasc Dis 2016; 25: 1655-1664

[63] Du J, Tian L, Liu W, u. a. Effects of repetitive transcranial magnetic stimulation on motor recovery and motor cortex excitability in patients with stroke: a randomized controlled trial. Eur J Neurol 2016; 23: $1666-1672$ 
[64] Li J, Meng X-M, Li R-Y, u. a. Effects of different frequencies of repetitive transcranial magnetic stimulation on the recovery of upper limb motor dysfunction in patients with subacute cerebral infarction. Neural Regen Res 2016; 11: 1584-1587

[65] Matsuura A, Onoda K, Oguro H, u.a. Magnetic stimulation and movement-related cortical activity for acute stroke with hemiparesis. Eur J Neurol 2015; 22: 1526-1532

[66] Lüdemann-Podubecká ], Bösl K, Theilig S, u.a. The Effectiveness of $1 \mathrm{~Hz}$ rTMS Over the Primary Motor Area of the Unaffected Hemisphere to Improve Hand Function After Stroke Depends on Hemispheric Dominance. Brain Stimul 2015; 8: 823-830

[67] Meng Z-Y, Song W-Q. Low frequency repetitive transcranial magnetic stimulation improves motor dysfunction after cerebral infarction. Neural Regen Res 2017; 12: 610-613

[68] Ameli M, Grefkes C, Kemper F, u. a. Differential effects of high-frequency repetitive transcranial magnetic stimulation over ipsilesional primary motor cortex in cortical and subcortical middle cerebral artery stroke. Ann Neurol 2009; 66: 298-309

[69] Long $\mathrm{H}$, Wang $\mathrm{H}$, Zhao C, u. a. Effects of combining high- and low-frequency repetitive transcranial magnetic stimulation on upper limb hemiparesis in the early phase of stroke. Restorative Neurology and Neuroscience 2018; 36: 21-30

[70] Duque J, Hummel F, Celnik P, u. a. Transcallosal inhibition in chronic subcortical stroke. Neurolmage 2005; 28: 940-946

[71] Nowak DA, Grefkes C, Dafotakis M, u. a. Effects of Low-Frequency Repetitive Transcranial Magnetic Stimulation of the Contralesional Primary Motor Cortex on Movement Kinematics and Neural Activity in Subcortical Stroke. Archives of Neurology 2008; 65: 741-747

[72] Grefkes C, Nowak DA, Wang LE, u. a. Modulating cortical connectivity in stroke patients by rTMS assessed with fMRI and dynamic causal modeling - ScienceDirect. Neurolmage 2010; 2013: 233-242 Im Internet: http://www.sciencedirect.com/science/article/pii/ S1053811909013172

[73] Fregni F, Boggio PS, Valle AC, u. a. A sham-controlled trial of a 5-day course of repetitive transcranial magnetic stimulation of the unaffected hemisphere in stroke patients. Stroke 2006; 37: 2115-2122

[74] Avenanti A, Coccia M, Ladavas E, u. a. Low-frequency rTMS promotes use-dependent motor plasticity in chronic stroke: A randomized trial. Neurology 2012; 78: 256-264

[75] Emara TH, Moustafa RR, EINahas NM, u. a. Repetitive transcranial magnetic stimulation at $1 \mathrm{~Hz}$ and $5 \mathrm{~Hz}$ produces sustained improvement in motor function and disability after ischaemic stroke. Eur J Neurol 2010; 17: 1203-1209

[76] Harvey RL, Edwards D, Dunning K, u. a. Randomized Sham-Controlled Trial of Navigated Repetitive Transcranial Magnetic Stimulation for Motor Recovery in Stroke. Stroke 2018; 49: 2138-2146

[77] Diekhoff-Krebs S, Pool E-M, Sarfeld A-S, u. a. Interindividual differences in motor network connectivity and behavioral response to iTBS in stroke patients. Neurolmage: Clinical 2017; 15: 559-571

[78] Hao Z, Wang D, Zeng Y, u. a. Repetitive transcranial magnetic stimulation for improving function after stroke. Cochrane Database of Systematic Reviews 2013; 78: 256-256

[79] Sattler V, Acket B, Raposo N, u. a. Anodal tDCS Combined With Radial Nerve Stimulation Promotes Hand Motor Recovery in the Acute Phase After Ischemic Stroke. Neurorehabilitation and Neural Repair 2014; 29: 743-754

[80] Khedr EM, Shawky OA, El-Hammady DH, u. a. Effect of Anodal Versus Cathodal Transcranial Direct Current Stimulation on Stroke Rehabilitation. Neurorehabilitation and Neural Repair 2013; 27: 592-601
[81] Chang MC, Kim DY, Park DH. Enhancement of Cortical Excitability and Lower Limb Motor Function in Patients With Stroke by Transcranial Direct Current Stimulation. Brain Stimul 2015; 8: 561-566

[82] Hesse S, Waldner A, Mehrholz J, u. a. Combined Transcranial Direct Current Stimulation and Robot-Assisted Arm Training in Subacute Stroke Patients. Neurorehabilitation and Neural Repair 2011; 25: 838-846

[83] Rossi C, Sallustio F, Di Legge S, u. a. Transcranial direct current stimulation of the affected hemisphere does not accelerate recovery of acute stroke patients. Eur J Neurol 2013; 20: 202-204

[84] Allman C, Amadi U, Winkler AM, u. a. Ipsilesional anodal tDCS enhances the functional benefits of rehabilitation in patients after stroke. Science Translational Medicine 2016; 8: 330re1

[85] Geroin C, Picelli A, Munari D, u. a. Combined transcranial direct current stimulation and robot-assisted gait training in patients with chronic stroke: a preliminary comparison. Clin Rehabil 2011; 25: 537-548

[86] Viana RT, Laurentino GEC, Souza RJP, u. a. Effects of the addition of transcranial direct current stimulation to virtual reality therapy after stroke: a pilot randomized controlled trial. NRE 2014; 34: 437-446

[87] Elsner B, Kugler J, Pohl M, u. a. Transcranial direct current stimulation (tDCS) for improving activities of daily living, and physical and cognitive functioning, in people after stroke. Cochrane Database of Systematic Reviews 2016; 96: S79-S190

[88] Naeser MA, Martin PI, Baker EH, u. a. Overt propositional speech in chronic nonfluent aphasia studied with the dynamic susceptibility contrast fMRI method. Neurolmage 2004; 22: 29-41

[89] Bhogal SK, Teasell R, Speechley M, Intensity of Aphasia Therapy, Impact on Recovery. Stroke 2003; 34: 987-993

[90] Thiel A, Hartmann A, Rubi-Fessen I, u. a. Effects of Noninvasive Brain Stimulation on Language Networks and Recovery in Early Poststroke Aphasia. Stroke 2013; 44: 2240-2246

[91] Waldowski K, Seniów ], Leśniak M., u. a. Effect of Low-Frequency Repetitive Transcranial Magnetic Stimulation on Naming Abilities in Early-Stroke Aphasic Patients: A Prospective, Randomized, DoubleBlind Sham-Controlled Study. The Scientific World Journal 2012; 2012: 1-8

[92] Heiss W-D, Hartmann A, Rubi-Fessen I, u. a. Noninvasive Brain Stimulation for Treatment of Right- and Left-Handed Poststroke Aphasics. Cerebrovasc Dis 2013; 36: 363-372

[93] Seniów ], Waldowski K, Leśniak M, u.a. Transcranial Magnetic Stimulation Combined with Speech and Language Training in Early Aphasia Rehabilitation: A Randomized Double-Blind Controlled Pilot Study. Topics in Stroke Rehabilitation 2015; 20: 250-261

[94] Rubi-Fessen I, Hartmann A, Huber W, u. a. Add-on Effects of Repetitive Transcranial Magnetic Stimulation on Subacute Aphasia Therapy: Enhanced Improvement of Functional Communication and Basic Linguistic Skills. A Randomized Controlled Study. Arch Phys Med Rehabil 2015; 96: e2

[95] Yoon TH, Han S], Yoon TS, u. a. Therapeutic effect of repetitive magnetic stimulation combined with speech and language therapy in post-stroke non-fluent aphasia. NRE 2015; 36: 107-114

[96] Hu X-Y, Zhang T, Rajah GB, u. a. Effects of different frequencies of repetitive transcranial magnetic stimulation in stroke patients with non-fluent aphasia: a randomized, sham-controlled study. Neurological Research 2018; 1-7

[97] Tsai P-Y, Wang C-P, Ko JS, U. a. The Persistent and Broadly Modulating Effect of Inhibitory rTMS in Nonfluent Aphasic Patients. Neurorehabilitation and Neural Repair 2014; 28: 779-787

[98] Wang C-P, Hsieh C-Y, Tsai P-Y, u. a. Efficacy of Synchronous Verbal Training During Repetitive Transcranial Magnetic Stimulation in Patients With Chronic Aphasia. Stroke 2014; 45: 3656-3662 
[99] Elsner B, Kugler J, Pohl M, u. a. Transcranial direct current stimulation (tDCS) for improving aphasia in adults with aphasia after stroke. Cochrane Database of Systematic Reviews 2019; 41: 1229-112

[100] Biou E, Cassoudesalle H, Cogné M, u. a. Transcranial direct current stimulation in post-stroke aphasia rehabilitation: A systematic review. Annals of Physical and Rehabilitation Medicine 2019; 62: 104-121

[101] de Aguiar V, Paolazzi CL, Miceli G. tDCS in post-stroke aphasia: the role of stimulation parameters, behavioral treatment and patient characteristics. Cortex 2015; 63: 296-316

[102] You DS, Kim DY, Chun MH, u. a. Cathodal transcranial direct current stimulation of the right Wernicke's area improves comprehension in subacute stroke patients. Brain and Language 2011; 119: 1-5

[103] Spielmann K, van de Sandt-Koenderman WME, Heijenbrok-Kal MH, u. a. Transcranial Direct Current Stimulation Does Not Improve Language Outcome in Subacute Poststroke Aphasia. Stroke 2018; 49: 1018-1020

[104] Polanowska KE, Leśniak MM, Seniów JB, u.a. Anodal transcrania direct current stimulation in early rehabilitation of patients with post-stroke non-fluent aphasia: a randomized, double-blind, sham-controlled pilot study. Restorative Neurology and Neuroscience 2013; 31: 761-771

[105] Fridriksson J, Rorden C, Elm J, u. a. Transcranial Direct Current Stimulation vs Sham Stimulation to Treat Aphasia After Stroke. JAMA Neurol 2018; 75: 1470-1477

[106] Fridriksson J, Richardson JD, Baker JM, u. a. Transcranial Direct Current Stimulation Improves Naming Reaction Time in Fluent Aphasia. Stroke 2011; 42: 819-821

[107] Baker JM, Rorden C, Fridriksson J. Using Transcranial Direct-Current Stimulation to Treat Stroke Patients With Aphasia. Stroke 2010; 41: $1229-1236$

[108] Fiori V, Coccia M, Marinelli CV, u. a. Transcranial direct current stimulation improves word retrieval in healthy and nonfluent aphasic subjects. Journal of Cognitive Neuroscience 2011; 23: 2309-2323

[109] Campana S, Caltagirone C, Marangolo P. Combining Voxel-based Lesion-symptom Mapping (VLSM) With A-tDCS Language Treatment: Predicting Outcome of Recovery in Nonfluent Chronic Aphasia. Brain Stimul 2015; 8: 769-776

[110] Yi YG, Chun MH, Do KH, u. a. The Effect of Transcranial Direct Current Stimulation on Neglect Syndrome in Stroke Patients. Ann Rehabil Med 2016; 40: 223-227

[111] Làdavas E, Giulietti S, Avenanti A, u. a. a-tDCS on the ipsilesional parietal cortex boosts the effects of prism adaptation treatment in neglect. Restorative Neurology and Neuroscience 2015; 33: 647-662

[112] Cazzoli D, Muri RM, Schumacher R, u. a. Theta burst stimulation reduces disability during the activities of daily living in spatial neglect. Brain 2012; 135: 3426-3439

[113] Koch G, Bonnì S, Giacobbe V, u. a. $\theta$-burst stimulation of the left hemisphere accelerates recovery of hemispatial neglect. Neurology 2012; 78: 24-30

[114] Nyffeler T, Vanbellingen T, Kaufmann BC, u. a. Theta burst stimulation in neglect after stroke: functional outcome and response variability origins. Brain 2019; 142: 992-1008

[115] Nyffeler T, Cazzoli D, Hess CW, u. a. One Session of Repeated Parietal Theta Burst Stimulation Trains Induces Long-Lasting Improvement of Visual Neglect. Stroke 2009; 40: 2791-2796
[116] Fu W, Song W, Zhang Y, u. a. Long-term effects of continuous theta-burst stimulation in visuospatial neglect. J Int Med Res 2015; 43: 196-203

[117] Bornheim S, Maquet P, Croisier JL, u. a. Motor cortex Transcranial Direct Current Stimulation (tDCS) improves acute stroke visuo-spatial neglect: A series of four case reports. Brain Stimul 2019; 11: 459-461

[118] Ko M-H, Han S-H, Park S-H, u. a. Improvement of visual scanning after DC brain polarization of parietal cortex in stroke patients with spatial neglect. Neuroscience Letters 2008; 448: 171-174

[119] Smit M, Schutter DJLG, Nijboer TCW, u. a. Transcranial direct current stimulation to the parietal cortex in hemispatial neglect: A feasibility study. Neuropsychologia 2015; 74: 152-161

[120] Sunwoo H, Kim Y-H, Chang WH, u. a. Effects of dual transcranial direct current stimulation on post-stroke unilateral visuospatial neglect. Neuroscience Letters 2013; 554: 94-98

[121] Tscherpel C, Hensel L, Lemberg K, u. a. The differential roles of contralesional frontoparietal areas in cortical reorganization after stroke. Brain Stimul 2020; 13: 614-624

[122] Lotze M, Markert J, Sauseng P, u. a. The role of multiple contralesional motor areas for complex hand movements after internal capsular lesion. Journal of Neuroscience 2006; 26: 6096-6102

[123] Johansen-Berg H, Rushworth MFS, Bogdanovic MD, u.a. The role of ipsilateral premotor cortex in hand movement after stroke. Proc Natl Acad Sci USA 2002; 99: 14518-14523

[124] Rehme AK, Volz LJ, Feis DL, u.a. Identifying Neuroimaging Markers of Motor Disability in Acute Stroke by Machine Learning Techniques. Cerebral Cortex 2015; 25: 3046-3056

[125] Rehme AK, Volz LJ, Feis D-L, u. a. Individual prediction of chronic motor outcome in the acute post-stroke stage: Behavioral parameters versus functional imaging. Hum Brain Mapp 2015; 36: 4553-4565

[126] Siegel JS, Seitzman BA, Ramsey LE, u. a. Re-emergence of modular brain networks in stroke recovery. Cortex 2018; 101: 44-59

[127] Zrenner C, Belardinelli P, Müller-Dahlhaus F, u. a. Closed-Loop Neuroscience and Non-Invasive Brain Stimulation: A Tale of Two Loops. Frontiers in Cellular Neuroscience 2016; 10: 92

[128] Zrenner C, Desideri D, Belardinelli P, u. a. Brain Stimulation. Brain Stimul 2018; 11: 374-389

[129] Hussain S], Hayward W, Fourcand F, u. a. Phase-dependent transcranial magnetic stimulation of the lesioned hemisphere is accurate after stroke. Brain Stimul 2020; 1-11

[130] Tscherpel C, Dern S, Hensel L et al. Brain responsivity provides an individual readout for motor recovery after stroke. Brain 2020; 143: 1873-1888

[131] Hensel L, Grefkes C, Tscherpel C, u. a. Intermittent theta burst stimulation applied during early rehabilitation after stroke: study protocol for a randomised controlled trial. BMJ Open 2019; 9 (12): e034088

[132] Kleineberg NN, Richter MK, Becker I, u. a. Verum versus sham tDCS in the treatment of stroke-induced apraxia: study protocol of the randomized controlled trial RAdiCS -"Rehabilitating (stroke-induced) Apraxia with direct Current stimulation”. Neurological Research and Practice 2020; $2: 7$ 